\title{
Essentiality, conservation, evolutionary pressure and codon bias in bacterial genes
}

\author{
Maddalena Dilucca, ${ }^{1}$ Giulio Cimini, ${ }^{2,3}$ and Andrea Giansanti ${ }^{1,4}$ \\ ${ }^{1}$ Dipartimento di Fisica, Sapienza University of Rome, Rome - Italy ${ }^{*}$ \\ ${ }^{2}$ IMT School for Advanced Studies, Lucca - Italy \\ ${ }^{3}$ Istituto dei Sistemi Complessi (ISC)-CNR, Rome - Italy \\ ${ }^{4}$ INFN Roma1 unit, Rome - Italy
}

\begin{abstract}
Essential genes constitute the core of genes which cannot be mutated too much nor lost along the evolutionary history of a species. Natural selection is expected to be stricter on essential genes and on conserved (highly shared) genes, than on genes that are either nonessential or peculiar to a single or a few species. In order to further assess this expectation, we study here how essentiality of a gene is connected with its degree of conservation among several unrelated bacterial species, each one characterised by its own codon usage bias. Confirming previous results on $E$. coli, we show the existence of a universal exponential relation between gene essentiality and conservation in bacteria. Moreover we show that, within each bacterial genome, there are at least two groups of functionally distinct genes, characterised by different levels of conservation and codon bias: i) a core of essential genes, mainly related to cellular information processing; ii) a set of less conserved nonessential genes with prevalent functions related to metabolism. In particular, the genes in the first group are more retained among species, are subject to a stronger purifying conservative selection and display a more limited repertoire of synonymous codons. The core of essential genes is close to the minimal bacterial genome, which is in the focus of recent studies in synthetic biology, though we confirm that orthologs of genes that are essential in one species are not necessarily essential in other species. We also list a set of highly shared genes which, reasonably, could constitute a reservoir of targets for new anti-microbial drugs.
\end{abstract}

\section{INTRODUCTION}

From an evolutionary point of view, all living species are in a process of adaptation to the environments they happen to live in. This process rests on the incorporation of genetic mutations into the genomes at the level of populations of species, which evolves on time-scales far longer than the time-scale of a generation. Signals from this process can be searched for in the sequences of single genes, of several genes within one single species, and among several species. In a previous work we have shown that, in E. coli, essentiality and degree of conservation of genes are subtly correlated with the codon bias displayed by their sequences [1]. In this work we extend those observations to a set of unrelated bacterial species, by elaborating on the connection between gene essentiality and conservation, and their relation with codon bias.

Individual genes in the genome of a given species contribute differentially to the survival and propagation of the organisms of that species. According to their known functional profiles and based on experimental evidences, genes can be divided into two categories: essential and nonessential ones 2, 3. Essential genes are not dispensable for the survival of an organism in the environment it lives in and the functions they encode are, therefore, considered as fundamental for life, irrespective of environmental changes [3, 4]. On the other hand, nonessential genes are those which are dispensable [5], being related to functions that can be silenced without lethal effects for the phenotype. Naturally, each species has adapted to one or more evolving environments and, plausibly, genes that are essential for one species may be not essential for another one. However, the set of genes that are essential in several bacterial species should encode for functions that are fundamental for life. As suggested by a quite broad literature, essential genes are more conserved than nonessential ones 6-10. It is worth noting that the term "conservation" has at least a twofold meaning. On the one hand, a gene is conserved if orthologous copies are found in the genomes of many species, as measured by the Evolutionary Retention Index (ERI) 2, 11. On the other hand, a gene is (evolutionarily) conserved when it is subject to a purifying evolutionary pressure which disfavors mutations [6, 12]. In this second meaning a conserved gene is, generically, a slowly evolving gene. The ratio $K_{a} / K_{s}$ of the number of nonsynonymous substitutions per nonsynonymous site to the number of synonymous substitutions per synonymous site is a widely used measure, though not exempt from criticism, to assess whether a gene is under Darwinian selection or a purifying evolutionary pressure.

Beyond essentiality and conservation, in our analysis we also consider the degeneracy of the genetic code, due to the fact that the same amino acid is encoded by different codon triplets (synonymous codons). Usage frequencies of synonymous codons vary significantly between different organisms, and also between proteins within the same organism 13. This phenomenon, known as codon usage bias, can be measured by various indices (see [14] for an overview); we use here statistical indicators such as the effective number of codons and the relative synonymous codon usage.

Our analysis reveals that those genes which are more conserved among bacterial species are also prone to be 
essential. Moreover, the codon usage in these conserved genes is, in general, more optimized than in less conserved genes. We have also shown that essential, conserved genes tend to be subject to a relatively more purifying evolutionary pressure. We argue that the set of genes with the highest degree of conservation (i.e., those with ERI $=1$ ) could include putative novel targets for novel anti bacterial strategies, as suggested with rather similar arguments by Dötsch et al. [15].

\section{MATERIALS AND METHODS}

\section{Bacterial genomes}

In this work we consider a set of 45 bacterial genomes from unrelated species, whose details are provided in Table [1 Nucleotide sequences from complete bacterial genomes were downloaded from the FTP server of the National Center for Biotechnology Information (NCBI) (ftp://ftp.ncbi.nlm.nih.gov/genomes/ archive/old_genbank/Bacteria/) [16]. Note that 31 of the 45 species we collected are also present in the dataset selected by Gerdes et al. [2] in their seminal paper on $E$. coli's essential genes.

\section{Conservation and essentiality}

We use the Evolutionary Retention Index (ERI) of Gerdes et al. 22 as a proxy for gene conservation. We compute the ERI of a gene as the fraction of genomes in Table I that have at least an ortholog of the given gene. A low ERI value means that a gene is specific, common to a small number of genomes, whereas, high ERI is a characteristic of highly shared, conserved, possibly universal genes.

In order to investigate gene essentiality we use the Database of Essential Genes (DEG), available at www. essentialgene.org [8]. DEG classifies a gene as either essential or nonessential on the basis of a combination of experimental evidence (null mutations or trasposons) and general functional considerations. DEG collects genomes from Bacteria, Archea and Eukarya, with different degrees of coverage [17, 18. Of the 45 bacterial genomes we have collected, only 24 are covered - in toto or partiallyby DEG, as indicated in Table I.

\section{Clusters of orthologous genes}

We use the database of orthologous groups of proteins (COGs), available at http://ncbi.nlm.nih.gov/COG/, for the functional annotation of gene sequences [19]. We consider 15 functional classes given by COGs, excluding the generic categories $\mathrm{R}$ and $\mathrm{S}$ for which functional annotation is too general or missing. Given a group of genes in a genome, we evaluate the conditional probability that these genes belong to a specific COG as:

$$
P(\mathrm{COG} \mid \text { group })=P(\text { group } \mid \mathrm{COG}) P(\mathrm{COG}) / P(\text { group }),
$$

where $P$ (group) is the size of the group with respect to the genome, $P(\mathrm{COG})$ is the fraction of the genome belonging to the $\mathrm{COG}$, and $P$ (group $\mid \mathrm{COG}$ ) is the fraction of genes in a given $\mathrm{COG}$ that belong to the group.

$$
\mathbf{K}_{a} / \mathbf{K}_{s}
$$

$K_{a} / K_{s}$ is the ratio of nonsynonymous substitutions per nonsynonymous site $\left(K_{a}\right)$ to the number of synonymous substitutions per synonymous site $\left(K_{s}\right)$ [12. This parameter is widely accepted as a straightforward and effective way of separating genes subject to purifying selection $\left(K_{a} / K_{s}<1\right)$ from genes subject to positive Darwinian selection $\left(K_{a} / K_{s}>1\right)$. There are different methods to evaluate this ratio, though the alternative approaches are quite consistent among themselves. For the sake of comparison, we have used here the $K_{a} / K_{s}$ estimates by Luo et al. [8] which are based on method by Nei and Gojobori 20. Note that each genome has a specific average level of $K_{a} / K_{s}$.

To study the patterns of $K_{a} / K_{s}$ in a given group of genes, we use Z-score values:

$$
Z_{g}\left[\left(K_{a} / K_{s}\right) \mid \text { group }\right]=\frac{\left\langle K_{a} / K_{s}\right\rangle_{\text {group }, g}-\left\langle K_{a} / K_{s}\right\rangle_{g}}{\sigma_{g} / \sqrt{N_{g}}},
$$

where $\left\langle K_{a} / K_{s}\right\rangle_{\text {group, } g}$ is the average of the ratio within a given group in a genome $g,\left\langle K_{a} / K_{s}\right\rangle_{g}$ and $\sigma_{g}$ are the average value of $K_{a} / K_{s}$ and its standard deviation over the whole genome $g$, and $N_{g}$ is the number of genes in the genome (we use the standard deviation of the mean as we are comparing average values). In the following we will analyze $K_{a} / K_{s}$ patterns with respect to group of genes with similar values of ERI, as indicated by $Z_{g}\left[\left(K_{a} / K_{s}\right) \mid \mathrm{ERI}\right]$ (eventually discerning essential and nonessential genes), as well as with respect to COGs, as indicated by $Z_{g}\left[\left(K_{a} / K_{s}\right) \mid \mathrm{COG}\right]$.

Note that $K_{a} / K_{s}$ values can differ much by magnitude not only among genes in different genomes but even for genes in the same genome, and their genome-specific distribution is rather broad with a high peak at zero (see below). In such a situation, larger values can in principle bias arithmetic averages. On the other hand, using alternative methods like the geometric mean, the arithmetic mean of the logarithms or the harmonic mean cannot be used because of the frequent zero values. Using the median and the median absolute deviation in a MannWhitney-Wilcoxon $U$ test instead of arithmetic means 
TABLE I. List of bacterial genomes. For each genome we report organism name, abbreviation, class, ncBI RefSeq, size (number of genes) and percentage of COG. Classes are: Alphaproteobacteria (1), Betaproteobacteria (2), Gammaproteobacteria (3), Epsilonproteobacteria (4), Actinobacteria (5), Bacilli (6), Bacteroidetes (7), Clostridia (8), Deinococci (9), Mollicutes (10), Spirochaetales (11), Aquificae (12), Cyanobacteria (13), Chlamydiae (14), Fusobacteria (15), Thermotoga (16). Asterisks denote genomes considered in [2]. For those genomes annotated in the Database of Essential Genes (DEG, highlighted in gray) we report the number of essential (E) and nonessential (NE) genes, as well as the coverage of essentiality.

\begin{tabular}{|c|c|c|c|c|c|c|c|c|}
\hline Organism & abbr. & class & ncBI RefSeq & size & $\% \mathrm{COG}$ & $\mathbf{E}$ & NE & cov. (\%) \\
\hline Agrobacterium tumefaciens (fabrum) & agtu* & 1 & nc_003062 & 2765 & 83.34 & & & \\
\hline Aquifex aeolicus VF5 & aqae* $^{*}$ & 12 & nc_000918 & 1497 & 86.65 & & & \\
\hline Bacillus subtilis 168 & basu* & 6 & nc_000964 & 4175 & 76.84 & 271 & 3904 & 100 \\
\hline Bacteroides thetaiotaomicron VPI-5482 & bath & 7 & nc_004663 & 4778 & 68.22 & 325 & 4453 & 100 \\
\hline Brucella melitensis bv. 1 str. $16 \mathrm{M}$ & brme* & 1 & nc_003317.1 & 2059 & 93.50 & & & \\
\hline Buchnera aphidicola Sg uid57913 & busg* & 3 & nc_004061 & 546 & 100 & & & \\
\hline Burkholderia pseudomallei K96243 & bups & 2 & nc_006350 & 3398 & 88.80 & 423 & 2932 & 98.8 \\
\hline Burkholderia thailandensis E264 & buth & 2 & nc_007651 & 3276 & 81.76 & 364 & 2912 & 100 \\
\hline Campylobacter jejuni & caje* & 4 & nc_002163 & 1572 & 82.49 & 222 & 1350 & 100 \\
\hline Caulobacter crescentus & cacr* & 1 & nc_011916 & 3885 & 65.55 & 402 & 2649 & 78.5 \\
\hline Chlamydia trachomatis D/UW-3/CX & $\operatorname{chtr}^{*}$ & 14 & nc_000117.1 & 894 & 71.75 & & & \\
\hline Clostridium acetobutylicum ATCC 824 & $\operatorname{clac}^{*}$ & 8 & nc_003030.1 & 3602 & 77.80 & & & \\
\hline Corynebacterium glutamicum ATCC 13032 & $\operatorname{cog} 1^{*}$ & 5 & nc_003450.3 & 2959 & 74.54 & & & \\
\hline Deinococcus radiodurans $\mathrm{R} 1$ & dera* & 9 & nc_001263.1 & 2629 & 72.86 & & & \\
\hline Escherichia Coli K-12 MG1655 & esco $^{*}$ & 3 & nc_000913.3 & 4004 & 86.98 & 587 & 2907 & 87.3 \\
\hline Francisella novicida U112 & frno & 3 & nc_008601 & 1719 & 82.71 & 390 & 1329 & 100 \\
\hline Fusobacterium nucleatum ATCC 25586 & funu* & 15 & nc_003454.1 & 1983 & 79.65 & & & \\
\hline Haemophilus influenzae Rd KW20 & hain* & 3 & nc_000907.1 & 1610 & 93.28 & 625 & 503 & 70 \\
\hline Helicobacter pylori 26695 & hepy* & 4 & Nc_000915.2 & 1469 & 76.90 & 305 & 1065 & 93.3 \\
\hline isteria monocytogenes EGD-e & $\operatorname{limo} *$ & 6 & nc_003210.1 & 2867 & 84.33 & & & \\
\hline Mesorhizobium loti MAFF303099 & melo* & 1 & nc_002678.2 & 6743 & 80.33 & & & \\
\hline Mycoplasma genitalium G37 & myge & 10 & nc_000908 & 475 & 80.84 & 378 & 94 & 99.37 \\
\hline Mycoplasma pneumoniae M129 & mypn* & 10 & nc_000912.1 & 648 & 68.62 & & & \\
\hline Mycoplasma pulmonis UAB CTIP & mypu & 10 & nc_002771 & 782 & 71.57 & 309 & 321 & 80.56 \\
\hline Mycobacterium tuberculosis H37Rv & mytu* & 5 & nc_000962.3 & 3936 & 74 & 592 & 2892 & 88.5 \\
\hline Neisseria gonorrhoeae FA 1090 uid57611 & nego* & 2 & nc_002946 & 1894 & 76.07 & & & \\
\hline Porphyromonas gingivalis ATCC 33277 & pogi & 7 & nc_010729 & 2089 & 65.46 & 463 & 1626 & 100 \\
\hline Pseudomonas aeruginosa UCBPP-PA14 & psae* & 3 & nc_008463 & 5892 & 82.97 & 335 & 4461 & 81.4 \\
\hline Ralstonia solanacearum GMI1000 & raso $^{*}$ & 2 & nc_003295.1 & 3436 & 81.22 & & & \\
\hline Rickettsia prowazekii str. Madrid E & ripr* & 1 & nc_000963.1 & 8433 & 87.76 & & & \\
\hline Salmonella enterica serovar Typhi & saen & 3 & nc_004631 & 4352 & 78.28 & 358 & 3992 & 99.96 \\
\hline Shewanella oneidensis MR-1 & shon & 3 & nc_004347 & 4065 & 69.68 & 402 & 1032 & 32.28 \\
\hline Sinorhizobium meliloti 1021 & sime* & 1 & nc_003047.1 & 3359 & 90.26 & & & \\
\hline Sphingomonas wittichii RW1 & spwi & 1 & nc_009511 & 4850 & 83.89 & 535 & 4315 & 100 \\
\hline Staphylococcus aureus N315 & stau* & 6 & nc_002745.2 & 2582 & 81 & 302 & 2280 & 100 \\
\hline Staphylococcus aureus ncTC 8325 & stau $_{-}$ & 6 & nc_007795 & 2767 & 71.25 & 345 & 2406 & 100 \\
\hline Streptococcus pneumoniae TIGR4 & stpn* & 9 & nc_003028.3 & 1814 & 85 & & & \\
\hline Streptococcus pyogenes MGAS5448 & stpy & 6 & nc_007297 & 1865 & 77.52 & 227 & 1337 & 83.86 \\
\hline Streptococcus pyogenes NZ131 & stpy & 6 & nc_011375 & 1700 & 80.45 & 241 & 1177 & 83.41 \\
\hline Streptococcus sanguinis & stsa & 6 & nc_009009 & 2270 & 79.94 & 218 & 2052 & 100 \\
\hline Synechocystis sp. PCC 6803 & sysp* & 13 & nc_000911.1 & 3179 & 76.96 & & & \\
\hline Thermotoga maritima MSB8 & thma* & 16 & nc_000853.1 & 1858 & 86.64 & & & \\
\hline Treponema pallidum Nichols & trpa* & 11 & nc_000919.1 & 1036 & 71.50 & & & \\
\hline Vibrio cholerae N16961 & vich* & 3 & nc_002505 & 2534 & 85 & 447 & 2079 & 99.68 \\
\hline Xylella fastidiosa 9a5c & xyfa* & 3 & nc_002488 & 2766 & 62.96 & & & \\
\hline
\end{tabular}

and standard deviations in Z-score tests to compare distributions leads to results which are highly consistent with what we obtained with Z-scores, though more noisy due to the vanishing of the median in many distributions.

\section{Codon bias}

There are several methods and indices to estimate the degree of codon usage bias in a gene. For an overview of current methods, their classification and rationale see 
Roth et al. 14. We use here two basic statistical indicators: the Number of Effective Codons $\left(N_{c}\right)$ and the Relative Synonymous Codon Usage $(R S C U)$.

$N_{c}$ measures of the effective diversity of the codons used to code a given protein 21. In principle, $N_{c}$ ranges from 20 (when just one single codon is used to code each one of the amino acids) to 61 (when the entire degeneracy of the genetic code is fully deployed, and each amino acid is coded by all its synonymous codons on an equal footing). Given a sequence of interest, the computation of $N_{c}$ starts from $F_{\alpha}$, a quantity defined for each family $\alpha$ of synonymous codons (one for each amino acid):

$$
F_{\alpha}=\sum_{k=1}^{m_{\alpha}}\left(\frac{n_{k \alpha}}{n_{\alpha}}\right)^{2},
$$

where $m_{\alpha}$ is the number of different codons in $\alpha$ (each one appearing $n_{1_{\alpha}}, n_{2_{\alpha}}, \ldots, n_{m_{\alpha}}$ times in the sequence) and $n_{\alpha}=\sum_{k=1}^{m_{\alpha}} n_{k_{\alpha}} . N_{c}$ then weights these quantities on a sequence:

$N_{c}=N_{s}+\frac{K_{2} \sum_{\alpha=1}^{K_{2}} n_{\alpha}}{\sum_{\alpha=1}^{K_{2}}\left(n_{\alpha} F_{\alpha}\right)}+\frac{K_{3} \sum_{\alpha=1}^{K_{3}} n_{\alpha}}{\sum_{\alpha=1}^{K_{3}}\left(n_{\alpha} F_{\alpha}\right)}+\frac{K_{4} \sum_{\alpha=1}^{K_{4}} n_{\alpha}}{\sum_{\alpha=1}^{K_{4}}\left(n_{\alpha} F_{\alpha}\right)}$

where $N_{s}$ is the number of families with one codon only and $K_{m}$ is the number of families with degeneracy $m$ (the set of 6 synonymous codons for leucine can be split into one family with degeneracy 2 , similar to that of phenylalanine, and one family with degeneracy 4 , similar to that of proline). In this paper we evaluate $N_{c}$ by using the implementation provided in DAMBE 5.0 [22].

The relative synonymous codon usage $\left(R S C U_{i}\right)$ of each codon $i$ is estimated as:

$$
R S C U_{i}=\frac{X_{i}}{\frac{1}{N_{i}} \sum_{j=1}^{n_{i}} X_{j}}
$$

where $X_{i}$ is the number of occurrences, either in a gene or in the whole genome, of codon $i$. The sum in the denominator runs over $n_{i}$, the degeneracy of the family of synonymous codons $i$ belongs to. For each codon $i$, its $R S C U_{i}$ is comprised between zero (no usage) and 1 (when only that codon is used among its synonymous alternatives). We evaluate these values by using DAMBE 5.0 [22.

The $R S C U$ values of the various codons can be grouped together as the 64 components (including the start codon ATG and the stop codons TAA, TAG and TGA - which are differently used by different species) of vectors which measure codon usage bias in a given bacterial species. To detect different patterns of codon usage between species we use heat maps drawn with CIMMiner (http://discover.nci. nih.gov/cimminer), and we cluster $R S C U$ vectors using Euclidean distances and the Average Linkage cluster algorithm.

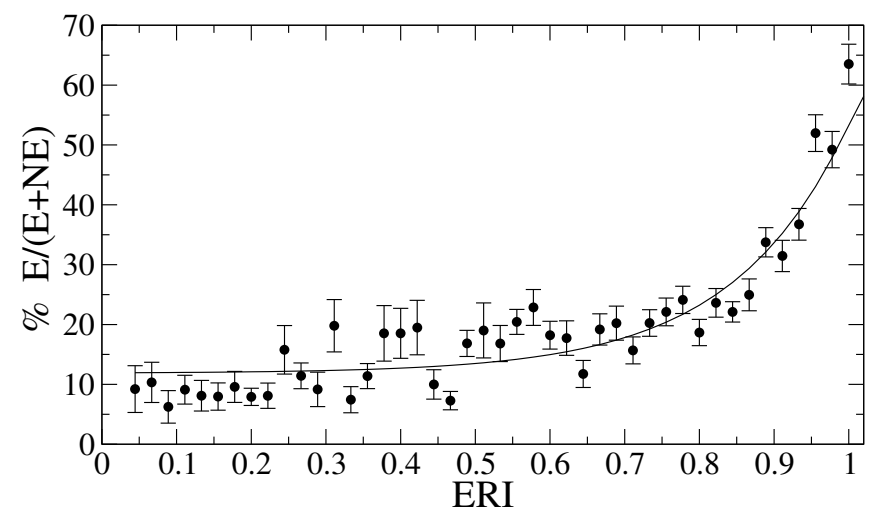

FIG. 1. Degree of essentiality versus ERI values. Genes from the 24 DEG-annotated genomes in Table I are aggregated into bins of ERI, and the fraction of essential genes in each bin is computed. Error bars are root mean square deviations, expressing the variability in percent essentiality from genome to genome. The solid line shows the exponential fit of the data $y=y_{0}+A \exp (B x)$, which returns $y_{0}=11 .(8), A=0.06(3)$, $B=6.4(8)$ and a coefficient of determination $R^{2}=0.90$. Note that since in small genomes essential genes outnumber nonessential ones (as we show below), we exclude from fitted data the two small genomes of myge and mypu. We also exclude stpn because it is poorly covered by DEG.

\section{RESULTS AND DISCUSSION}

\section{Essentiality and conservation in bacterial genes}

Figure 1 shows the percentage of essential genes within genes with a given value of ERI (which we recall operationally encodes the degree of conservation of a gene). The observed exponential dependence generalises to several unrelated species a basic result on E. coli, by Gerdes et al. 2] (see Figure 3 therein), and the fit parameters we find are strictly consistent with those reported in that paper. This points to the existence of a universal exponential correlation between gene essentiality and conservation in bacteria. Indeed, the fact that essential genes should be more evolutionarily conserved than nonessential ones has been previously shown, following different approaches [7, 23. Our result confirms those earlier observations and leads to conclude that the more a gene is shared, the more it is likely to be essential. This point will be further investigated in the next section.

Figure 2 further shows that the number of essential genes is rather constant among bacterial genomes. In small genomes with less than 1000 genes, most genes are essential. Then, as the size of the genome increases, the number of nonessential genes increases proportionally. Note that shon does not follow the trend: it is a species with a peculiar metabolism and, at present, is poorly covered by DEG. Independently from the genome size, each bacterial species has a core of about 400 essential genes. This observation can be related to recent 


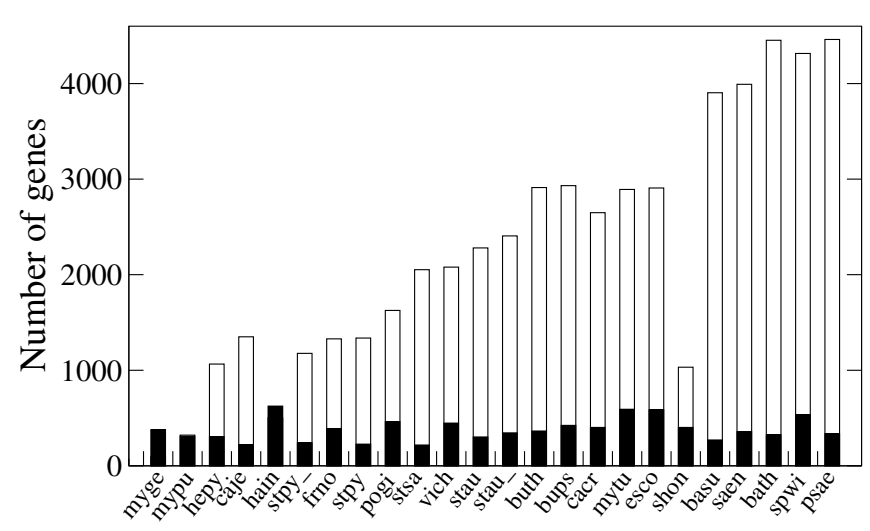

FIG. 2. Essential and nonessential genes in each bacteria. On the horizontal axis, DEG-annotated species from Table I are sorted according to the size of their genome. Black and white bars represent the number of essential and nonessential genes. The number of essential genes is basically constant in all species (average value $=378 \pm 115$ ), while the number of nonessential genes increases with the size of the genomes.

experiments in synthetic biology, devoted to the in vitro assembly of artificial bacteria with minimal genomes, limited to those genes which are necessary to sustain basic life processes [24 26]. In particular, the synthetic bacterium designed and synthesized by Hutchison et al. [26] has a genome constituted by 473 genes from Mycoplasma mycoides, a species whose genome contains 475 genes and which is evolutionarily close to the Mycoplasma genitalium considered here (myge). Of the genes in myge, $80 \%$ are annotated as essential and the remaining $20 \%$ have no annotation yet; clearly there are still unknown functions that could be, nevertheless, essential for life.

It is tempting to suppose that the core of essential genes in the bacterial species of Figure 2 constitutes a kind of minimal, universal and conserved genome, made by genes that have an orthologous in all species. But this is not the case. We have checked that only 83 genes are strictly retained $(\mathrm{ERI}=1)$ among all the DEG-annotated bacterial species we consider (and are reported in Table II . Among them, no one is essential in all species, but only in a fraction $f(\mathrm{E})$ of the bacteria. Thus, essentiality does not strictly imply orthology: genes that are essential for one species may be not essential for another one. Indeed, the experimentally determined essentiality might disagree between species for a variety of reasons, for instance because experimental conditions that are near-optimal for one species are instead demanding for another species. Concerning strictly retained genes in general, as shown in Table III they have a quite restricted repertoire of functions, limited to COGs J (translation, ribosomal structure and biogenesis: 49 cases), K (transcription: 7 cases), L (replication, recombination and repair: 7 cases) and $\mathrm{O}$ (Post translational modification, protein turnover, chaperones: 8 cases). Hence, more than half of these genes correspond to ribosomal proteins with different degree of shared essentiality, as evaluated by $f(\mathrm{E})$. We have checked in DrugBank that several of these genes, as expected, are targets of antimicrobial drugs in E. coli, as shown with bold COG ids in Table [II] and we note that all these targets have a shared essentiality of at least 0.56 . It is then tempting to suppose that the set of strictly retained genes is a reservoir of highly druggable genes, characterised both by highly shared orthology and essentiality, to be further exploited in the design of next generation antimicrobial drugs [27]. This result somehow specialises what Luo et al. [8] found on the same set of bacterial species: "essential genes in the functional COG categories G, H, I, J, K and L tend to be more evolutionarily conserved than the corresponding nonessential genes in bacteria". This kind of general statement deserves more investigation. First of all, in the next section we consider how essential and nonessential genes are partitioned into different COGs.

\section{Functional specialization of essential and nonessential genes}

The heat maps of Figure 3 represent conditional probabilities $P(\mathrm{COG} \mid \mathrm{E})$ and $P(\mathrm{COG} \mid \mathrm{NE})$ that essential and nonessential genes belong to the different COGs, for the various bacterial species we consider. Essential and nonessential genes have different functional spectra. In both panels, a banded vertical structure emerges which roughly separates COGs into three groups. In particular, $51 \%$ of essential genes fall into $\mathrm{J}, \mathrm{M}, \mathrm{H}$ and $\mathrm{L}$, whereas $49 \%$ of nonessential genes belong to E, K, G, P and C. Table III synthetically shows that essential genes dominate functions related to information storage and processing, whereas nonessential genes prevail among the set of functions related to metabolism. Functions related to cellular processes and signalling appear to be equally shared between essential and nonessential genes. In the next section, using the criteria of the $K_{a} / K_{s}$ ratio, we challenge the sensible statement that essential genes are subject to a stricter purifying selection than nonessential genes. If that were true, then each COG would exhibit a signature of either purifying or positive selection, on the basis of the fraction of essential genes that belong to it.

\section{Selective pressure, conservation and essentiality}

In this section we firstly consider how evolutionary pressure, as represented by the ratio $K_{a} / K_{s}$, correlates with the degree of retention (conservation) of bacterial genes. Note that each bacterial genome has its own level of evolutionary pressure (see Figures 4 and 10 . We thus compare, within each genome, the evolutionary pressure that is exerted over more or less conserved genes. Using the thresholds of ERI used in Dilucca et al. [1], Figure 4 
TABLE II. List of strictly retained genes $(\mathbf{E R I}=1)$. These 83 genes have orthologous copies in all the 45 bacteria of Table I] and are sorted by Z-score values of $K_{a} / K_{s} . f(\mathrm{E})$ and $f(\mathrm{NE})$ is the fraction of genomes in which each gene is annotated as essential and nonessential, respectively, and $f(\mathrm{E})+f(\mathrm{NE})=1$ only when the gene is fully annotated. Note the prevalence of COG J. In bold we report the genes which are antibiotic drug targets in E. coli(see https://www.drugbank.ca/).

\begin{tabular}{|c|c|c|c|c|c|}
\hline COG ID & Gene & Protein name & $f(E)$ & $f(N E)$ & Z-score \\
\hline COG0238J & rpsR & $30 \mathrm{~S}$ ribosomal protein $\mathrm{S} 18$ & 0.60 & 0.28 & -18.79 \\
\hline COG0197J & $\mathrm{rplP}$ & 50 S ribosomal protein L 16 & 0.80 & 0.16 & -17.64 \\
\hline COG0080J & $\mathrm{rplK}$ & 50 S ribosomal protein L11 & 0.76 & 0.12 & -16.44 \\
\hline COG0250K & - & transcription antitermination protein NusG & 0.52 & 0.32 & -16.13 \\
\hline COG0185J & rpsS & 30 S ribosomal protein S19 & 0.68 & 0.20 & -15.80 \\
\hline COG0100J & rpS11 & 30 S ribosomal protein S11 & 0.76 & 0.12 & -15.47 \\
\hline COG0199J & $\mathrm{rpsN}$ & 30 S ribosomal protein $\mathrm{S} 14$ & 0.64 & 0.24 & -15.09 \\
\hline COG0090J & rplB & 50 S ribosomal protein L2 & 0.80 & 0.12 & -14.84 \\
\hline COG0202K & rpoA & DNA-directed RNA polymerase subunit alpha & 0.80 & 0.12 & -14.72 \\
\hline COG0093J & rplN & 50 S ribosomal protein L14 & 0.72 & 0.16 & -14.62 \\
\hline COG0092J & $\mathrm{rpsC}$ & 30 S ribosomal protein S3 & 0.88 & 0.04 & -14.44 \\
\hline COG0081J & rpla & 50 S ribosomal protein $\mathrm{L} 1$ & 0.64 & 0.24 & -14.19 \\
\hline COG0049J & rpS7 & 30 S ribosomal protein $\mathrm{S} 7$ & 0.88 & 0.08 & -13.90 \\
\hline COG0203J & rplQ & 50 S ribosomal protein $\mathrm{L} 17$ & 0.64 & 0.24 & -13.74 \\
\hline COG0211J & rpmA & 50 S ribosomal protein L27 & 0.72 & 0.12 & -13.74 \\
\hline COG0255J & $\mathrm{rpmC}$ & 50 S ribosomal protein L29 & 0.72 & 0.20 & -13.66 \\
\hline COG0103J & rpsI & 30 S ribosomal protein 59 & 0.56 & 0.32 & -13.20 \\
\hline COG0231J & efp & elongation factor $\mathrm{P}$ & 0.48 & 0.48 & -12.75 \\
\hline COG0691O & smpB & SsrA-binding protein & 0.28 & 0.52 & -12.21 \\
\hline COG0201U & $\sec Y$ & preprotein translocase subunit $\mathrm{Sec} Y$ & 0.84 & 0.08 & -12.18 \\
\hline COG0186J & rpsQ & $30 \mathrm{~S}$ ribosomal protein $\mathrm{S} 17$ & 0.72 & 0.16 & -10.59 \\
\hline COG0050J & tuf & elongation factor $\mathrm{Tu}$ & 0.48 & 0.36 & -10.43 \\
\hline COG0184J & $\mathrm{rpsO}$ & 30 S ribosomal protein $\mathrm{S} 15$ & 0.52 & 0.36 & -9.96 \\
\hline COG0305L & dnaB & replicative DNA helicase & 0.80 & 0.16 & -9.07 \\
\hline COG0336J & $\operatorname{trmD}$ & tRNA (guanine-N(1)-)-methyltransferase & 0.60 & 0.28 & -9.07 \\
\hline COG0261J & $\mathrm{rplU}$ & 50 S ribosomal protein L 21 & 0.56 & 0.28 & -8.87 \\
\hline COG0858J & - & ribosome-binding factor A & 0.40 & 0.36 & -8.70 \\
\hline COG0781K & - & transcription termination/antitermination protein NusB & 0.48 & 0.40 & -8.46 \\
\hline COG0097J & $\mathrm{rplF}$ & $50 S$ ribosomal protein L6 & 0.88 & 0.08 & -7.18 \\
\hline COG0222J & rplL & 50 S ribosomal protein $\mathrm{L} 7 / \mathrm{L} 12$ & 0.68 & 0.20 & -6.97 \\
\hline COG0088J & rplD & 50 S ribosomal protein $\mathrm{L} 4$ & 0.84 & 0.04 & -6.33 \\
\hline COG0089J & rplW & 50 S ribosomal protein L23 & 0.72 & 0.20 & -5.60 \\
\hline COG0091J & rplV & 50 S ribosomal protein L22 & 0.72 & 0.20 & -5.58 \\
\hline COG0087J & rplC & $50 \mathrm{~S}$ ribosomal protein $\mathrm{L} 3$ & 0.84 & 0.08 & -5.26 \\
\hline COG0541U & ffh & signal recognition particle protein & 0.80 & 0.12 & -4.82 \\
\hline COG0592L & dnaN & DNA polymerase III subunit beta & 0.72 & 0.16 & -4.47 \\
\hline COG0236IQ & - & acyl carrier protein & 0.48 & 0.32 & -3.67 \\
\hline COG0480J & fus & elongation factor $G$ & 0.56 & 0.40 & -2.87 \\
\hline COG0233J & frr & ribosome recycling factor & 0.88 & 0.12 & -2.80 \\
\hline COG0335J & rpls & 50 S ribosomal protein L19 & 0.56 & 0.32 & -2.48 \\
\hline COG0200J & rplo & 50 S ribosomal protein L 15 & 0.72 & 0.16 & -2.41 \\
\hline COG0084L & - & TatD family deoxyribonuclease & 0.16 & 0.80 & -2.19 \\
\hline COG0264J & tsf & elongation factor Ts & 0.80 & 0.12 & -2.15 \\
\hline COG0528F & pyrH & uridylate kinase & 0.84 & 0.16 & -1.42 \\
\hline COG0525J & vals & valyl-tRNA synthetase & 0.84 & 0.08 & -1.07 \\
\hline COG0359J & rplI & 50 S ribosomal protein L9 & 0.24 & 0.68 & -0.42 \\
\hline COG1136V & -8 & ABC transporter ATP-binding protein & 0.20 & 0.76 & -0.20 \\
\hline COG1136V & - & ABC transporter ATP-binding protein & 0.20 & 0.76 & -0.20 \\
\hline COG1136V & - & ABC transporter ATP-binding protein & 0.20 & 0.76 & -0.20 \\
\hline COG0172J & $\operatorname{serS}$ & seryl-tRNA synthetase & 0.84 & 0.12 & 0.29 \\
\hline COG0012J & - & GTP-dependent nucleic acid-binding protein EngD & 0.08 & 0.84 & 0.59 \\
\hline COG0571K & rnc & 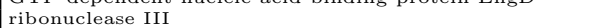 & 0.24 & 0.60 & 1.13 \\
\hline COG0563F & adk & adenylate kinase & 0.64 & 0.32 & 1.39 \\
\hline COG0244J & rplJ & 50 S ribosomal protein L10 & 0.72 & 0.16 & 1.71 \\
\hline COG0522J & rpsD & 30 S ribosomal protein S4 & 0.80 & 0.12 & 2.03 \\
\hline COG0188L & gyra & DNA gyrase subunit A & 0.64 & 0.24 & 2.27 \\
\hline COG0188L & gyr A & DNA gyrase subunit A & 0.64 & 0.24 & 2.27 \\
\hline COG0086K & rpoC & DNA-directed RNA polymerase subunit beta & 0.76 & 0.20 & 2.36 \\
\hline COG0180J & $\operatorname{trpS}$ & tryptophanyl-tRNA synthetase & 0.72 & 0.20 & 2.61 \\
\hline COG0629L & ssb & single-strand binding protein family & 0.48 & 0.44 & 2.65 \\
\hline COG0195K & nusA & transcription elongation factor NusA & 0.80 & 0.12 & 2.94 \\
\hline COG0013J & alas & alanyl-tRNA synthetase & 0.68 & 0.28 & 3.70 \\
\hline COG0228J & rpsP & $30 \mathrm{~S}$ ribosomal protein $\mathrm{S} 16$ & 0.64 & 0.28 & 3.88 \\
\hline COG0102J & rplM & 50 S ribosomal protein L13 & 0.80 & 0.04 & 4.57 \\
\hline COG0568K & rpoD & RNA polymerase sigma factor & 0.64 & 0.36 & 4.58 \\
\hline COG0072J & pheT & phenylalanyl-tRNA synthetase subunit beta & 0.76 & 0.20 & 4.75 \\
\hline COG0544O & tig & trigger factor & 0.00 & 0.88 & 5.11 \\
\hline COG0209F & nrdE & ribonucleotide-diphosphate reductase subunit alpha & 0.64 & 0.32 & 5.34 \\
\hline COG0484O & - & DnaJ domain-containing protein & 0.40 & 0.56 & 5.46 \\
\hline COG0484O & - & DnaJ domain-containing protein & 0.40 & 0.56 & 5.46 \\
\hline COG0484O & & DnaJ domain-containing protein & 0.40 & 0.56 & 5.46 \\
\hline COG0443O & dnaK & molecular chaperone DnaK & 0.60 & 0.36 & 5.82 \\
\hline COG0442J & pros & prolyl-tRNA synthetase & 0.92 & 0.08 & 5.91 \\
\hline COG0018J & $\operatorname{argS}$ & arginyl-tRNA synthetase & 0.72 & 0.20 & 9.22 \\
\hline COG0215J & cysS & cysteinyl-tRNA synthetase & 0.88 & 0.08 & 9.42 \\
\hline COG0536R & obgE & GTPase ObgE & 0.68 & 0.24 & 11.84 \\
\hline COG0552U & ftsY & signal recognition particle-docking protein FtsY & 0.80 & 0.12 & 12.44 \\
\hline COG05760 & $\operatorname{grpE}$ & co-chaperone GrpE & 0.68 & 0.24 & 13.17 \\
\hline COG0143J & mets & methionyl-tRNA synthetase & 0.64 & 0.32 & 14.64 \\
\hline COG1214O & - & glycoprotease & 0.60 & 0.32 & 15.12 \\
\hline COG0587L & polC-2 & DNA polymerase III subunit alpha & 0.72 & 0.24 & 20.79 \\
\hline COG0223J & fmt & methionyl-tRNA formyltransferase & 0.68 & 0.24 & 25.68 \\
\hline COG0313R & & tetrapyrrole (corrin/porphyrin) methylase protein & 0.08 & 0.76 & 26.42 \\
\hline
\end{tabular}

shows that more conserved genes (with ERI $>0.6$ ) significantly display lower values of $K_{a} / K_{s}$ than less conserved genes (with ERI $<0.2$ ). Interestingly, genes belonging to the core of 83 strictly conserved genes of ERI $=1$, mentioned above, have levels of $K_{a} / K_{s}$ that are systematically below the average value of the more conserved genes. This last observation stresses once more that the most conserved genes, those involved in more basic and universal functions, tend to be subject to a relatively purifying, conservative selection. Since highly conserved genes are also prone to be essential, as shown in Figure 1, our observation confirms the previous conclusion by Luo et al. [8] that "essential genes are more evolutionarily conserved (they are characterised by a significantly lower 
TABLE III. Functional specialization of essential and nonessential genes according to COG clusters. Figures indicate the percentages of essential and nonessential genes within a given COG (sums of these figures for table subsections are reported in boldface). COGs are sorted by percent essentiality.

\begin{tabular}{clrr}
\hline \hline COG ID Functional classification & \% E & \% NE \\
\hline J & INFORMATION STORAGE AND PROCESSING & & \\
K & Translation, ribosomal structure and biogenesis & 0.25 & 0.05 \\
K & Transcription & 0.06 & 0.10 \\
L & Replication, recombination and repair & 0.08 & 0.07 \\
& & $\mathbf{0 . 3 9}$ & $\mathbf{0 . 2 2}$ \\
\hline & CELLULAR PROCESSES AND SIGNALING & & \\
D & Cell cycle control, cell division, chromosome partitioning & 0.03 & 0.01 \\
T & Signal transduction mechanisms & 0.02 & 0.07 \\
M & Cell wall/membrane/envelope biogenesis & 0.10 & 0.08 \\
N & Cell motility & 0.01 & 0.03 \\
O & Posttranslational modification, protein turnover, chaperones & 0.04 & 0.05 \\
& & $\mathbf{0 . 2 0}$ & $\mathbf{0 . 2 4}$ \\
\hline & METABOLISM & 0.07 & 0.08 \\
C & Energy production and conversion & 0.06 & 0.10 \\
G & Carbohydrate transport and metabolism & 0.06 & 0.12 \\
E & Amino acid transport and metabolism & 0.05 & 0.03 \\
F & Nucleotide transport and metabolism & 0.08 & 0.06 \\
H & Coenzyme transport and metabolism & 0.07 & 0.05 \\
I & Lipid transport and metabolism & 0.03 & 0.08 \\
P & Inorganic ion transport and metabolism & $\mathbf{0 . 4 1}$ & $\mathbf{0 . 5 2}$ \\
& $\quad$ & &
\end{tabular}

$\left.K_{a} / K_{s}\right)$ than nonessential ones in most of the bacteria".

Looking for a general relationship between the evolutive pressure exerted on a gene and its degree of conservation as measured by the ERI, in Figure 5 we show that when the degree of retention increases, the Z-score of $K_{a} / K_{s}$ systematically decreases, becoming more and more negative. This observation stresses again that those genes which are common to several species are subject to a purifying, more constrained evolution. Note that, comparatively, essential genes systematically have a Z-score which is more negative than for nonessential genes, indicating that they are, for each degree of retention, subject to a more purifying evolution.

Getting to the functional annotation provided by COGs, Luo et al. 8] also show that essential genes in each of the COGs G, H, I, J, K and L tend to be significantly more evolutionarily conserved than nonessential genes belonging to the same COGs. It would be then natural to conclude that the nature of the evolutionary pressure that is exerted on the genes belonging to a COG depends on its content of essential genes. From Table III it is possible to rank bacterial COGs and their functions by their content of essential genes. In particular, note that COGs J, M, H and L contain more than $51 \%$ of the annotated essential genes, and a recent experiment has re-confirmed in basu that precisely these COGs are enriched in essential genes (see Figure 2 in Koo et al. 28]). One would then conclude that the genes in these COGs should be under a more conservative evolutionary pressure than those belonging to the rest of the COGs. To elucidate this point, we evaluated the Z-scores of $K_{a} / K_{s}$ over the genes of each $\mathrm{COG}$ with respect to the average value of this ratio over the genomes they come from (re- sults in Table IV and in Figure 6). According to this analysis, one would conclude that the rank order of the evolutionary pressure on the COGs would be J, F, K, O, E, I, D, C, T, H, G, P, L, N, M, going from relatively purifying to more diversifying selection. In the first four ranks we find J, F, K and O, at variance with the expectation based on the content of essential genes.

From one point of view one could think that the observed discrepancy between the ranking based on the Zscore and the one based on the essentiality content should depend on the limited coverage of the available dataset. At present not all genes in the genomes we have investigated are annotated for essentiality and, even-worse, not all the genes have been attributed to a COG class (see Table I to check for the coverage of the essentiality and COG annotation). From another point of view, our Z-score statistics in Figure 6 is based on a set of 39.804 genes (annotated for $K_{a} / K_{s}$ and COG) over a total of 127.012. We believe our Z-score statistics is sufficiently representative of the overall evolutionary pressure exerted over the COG classes. On the basis of the data in Figure 6, we propose a tentative distinction between a set of relatively more evolutionarily conserved COGs (J, F, $\mathrm{K}$, and $\mathrm{O}$ ) and, on the other side, a set of more adaptive ones (P, L, N, and M). This distinction should be further tested, along with the progressive annotation of bacterial genomes. Notably COG J, the set of genes which is more exhaustively annotated, has the highest percentage of essential genes and with little uncertainty is, in all genomes, under a conservative evolutionary pressure.

To conclude this section we focus on the set of 83 genes which are highly retained in all species (those with ERI $=1$ ) and which display a restricted set of functional 

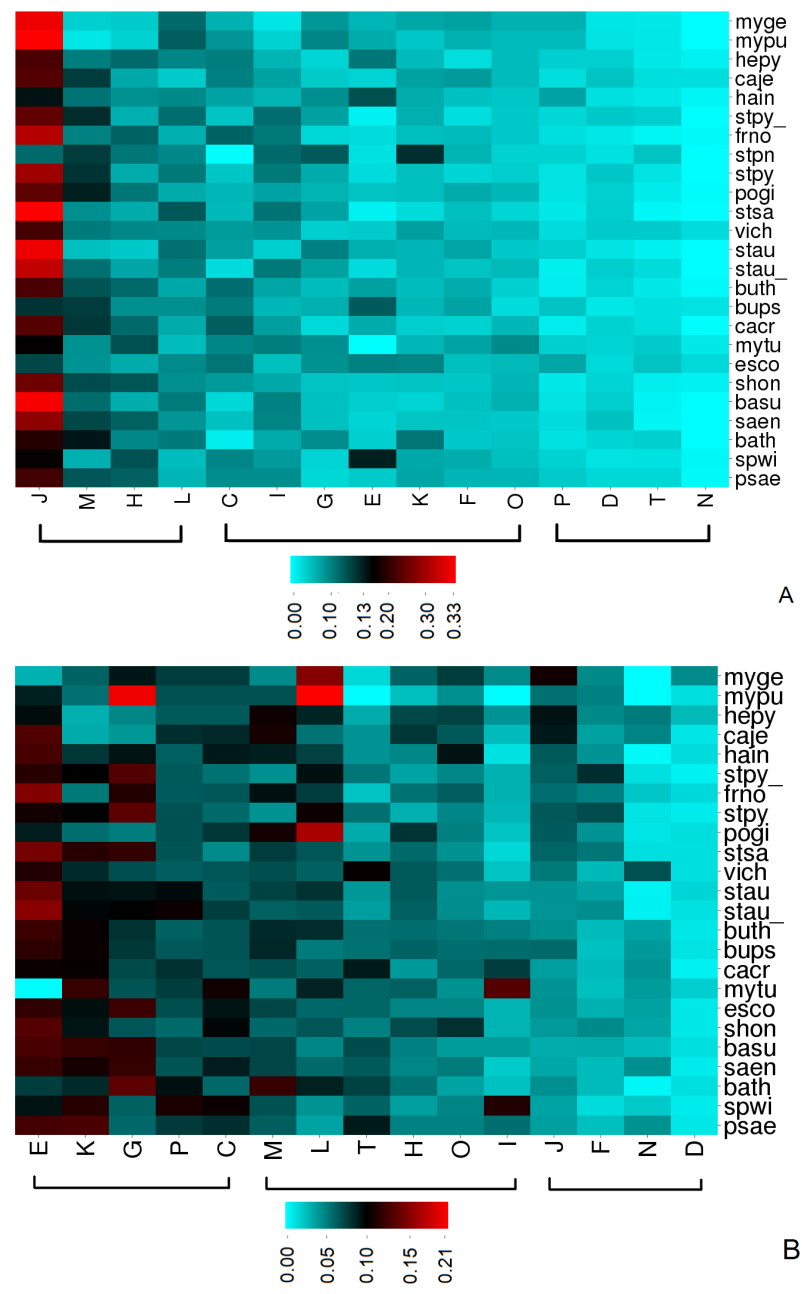

FIG. 3. For each genome we estimated the conditional probabilities $P(\mathrm{COG} \mid \mathrm{E})$ (panel $\mathrm{A}$ ) and $P(\mathrm{COG} \mid \mathrm{NE})$ (panel $\mathrm{B}$ ) for an essential and a nonessential gene to belong to a given $\mathrm{COG}$. Genomes are ranked from top to bottom by the size of their genomes. COGs are ranked, separately in both panels and from left to right, according to their overall incidence. In panel A, $51 \%$ of the essential genes belong, in different proportions, to COGs J, M, H and L; $40 \%$ to C, I, G, E, K, F and $\mathrm{O}$ and the remaining $10 \%$ to $\mathrm{P}, \mathrm{D}, \mathrm{T}$ and $\mathrm{N}$. In panel $\mathrm{B}$, $49 \%$ of the nonessential genes belong to $\mathrm{E}, \mathrm{K}, \mathrm{G}, \mathrm{P}$, and C; $38 \%$ to $\mathrm{M}, \mathrm{L}, \mathrm{T}, \mathrm{H}, \mathrm{O}$ and $\mathrm{I}$; the remaining $13 \%$ to $\mathrm{J}, \mathrm{F}, \mathrm{N}$, and D.

specialisations. In Figure 7 we show the histogram of the $K_{a} / K_{s}$ values for this core of genes in all DEG-annotated species of Table I] The plot indicates that even the genes with orthologous copies in all the genomes here considered rarely have values of $K_{a} / K_{s}$ bigger than 1 . This shows that they generally are under an overall purifying selection. It is worth to note that this distribution of $K_{a} / K_{s}$ is consistent with the distributions of the same parameter over the different COGs. This last observation points out that a sufficiently large set of bacterial genes display a similar distribution of $K_{a} / K_{s}$, which in

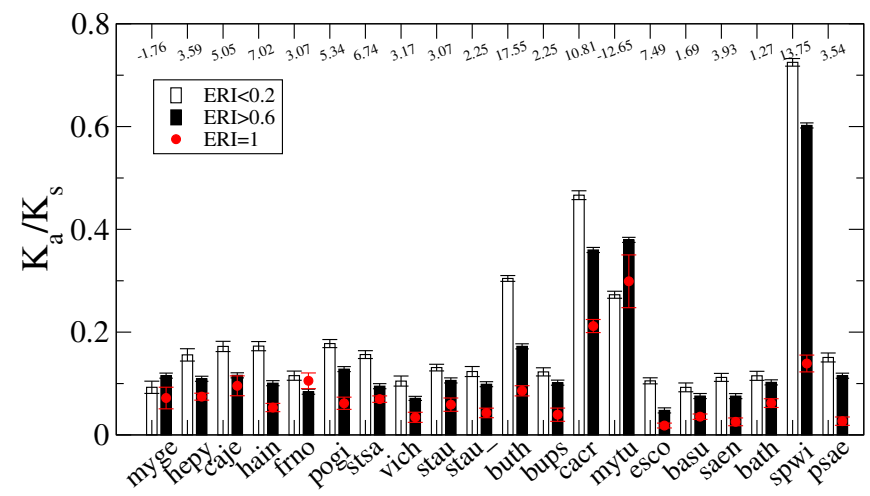

FIG. 4. Average values and standard deviations of $K_{a} / K_{s}$ for specific and conserved genes in each genome. Specific genes have ERI $<0.2$ and conserved genes have ERI $>0.6$. According to a two-sample $\mathrm{z}$ statistic, whose values are shown at the top of the panel, with the exception of myge and mytu, the average value of $K_{a} / K_{s}$ is significantly higher for specific genes. Red points denote averages and errors of $K_{a} / K_{s}$ for the most conserved genes (those with ERI $=1$ ).

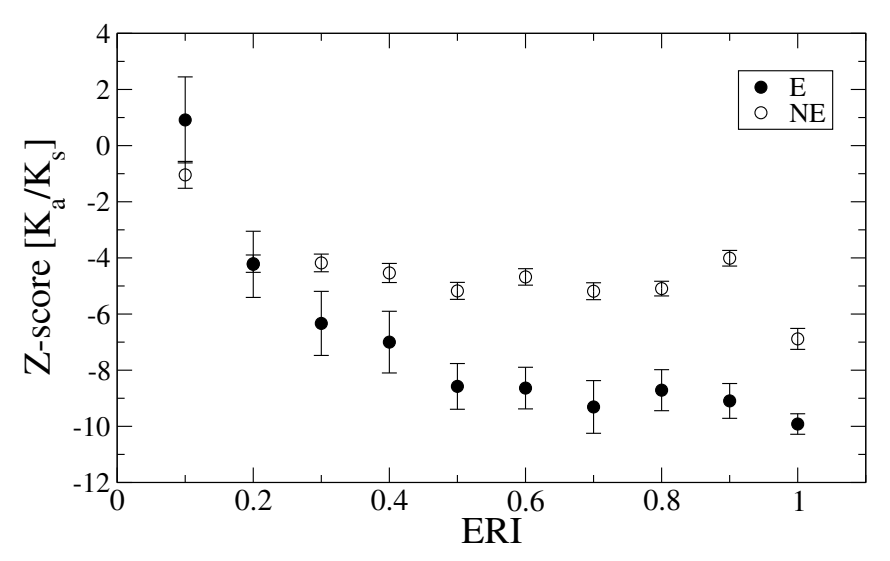

FIG. 5. Z-scores of $K_{a} / K_{s}$, relative to the average value in the species, for essential and nonessential genes within binned ERI values, for the DEG-annotated genomes of TableI Error bars are root mean square deviations for the genes falling in each bin. Z-scores decrease with the degree of conservation: the more a gene is retained among different species, the more is subject to a purifying selection. The two trends are well separated (with the exception of less conserved genes): average Z-scores of essential genes are systematically lower than those of nonessential genes, confirming that essential genes are subject to a more purifying, conservative evolutionary pressure.

turn indicates that in general these genes are subject to an overall purifying selection $\left(K_{a} / K_{s}<1\right)$. Nevertheless, through the relative comparison of the individual Z-scores of genes in different genomes and in different COGs, we can sensibly assess that the different COGs are under diverse evolutive pressure and constraints. 
TABLE IV. Ranking of COGs according to Z-scores of $\mathbf{K}_{\mathbf{a}} / \mathbf{K}_{\mathbf{s}}$. We count \pm 1 for each time the Z-score is smaller than -1 or bigger than +1 , respectively.

\begin{tabular}{|l|c|c|c|c|c|c|c|c|c|c|c|c|c|c|c|}
\hline COG ID & J & F & K & O & E & I & D & C & T & H & G & P & L & N & M \\
\hline Z-score & -20 & -19 & -14 & -13 & -13 & -13 & -12 & -12 & -9 & -9 & -6 & -5 & -5 & 4 & 5 \\
\hline
\end{tabular}

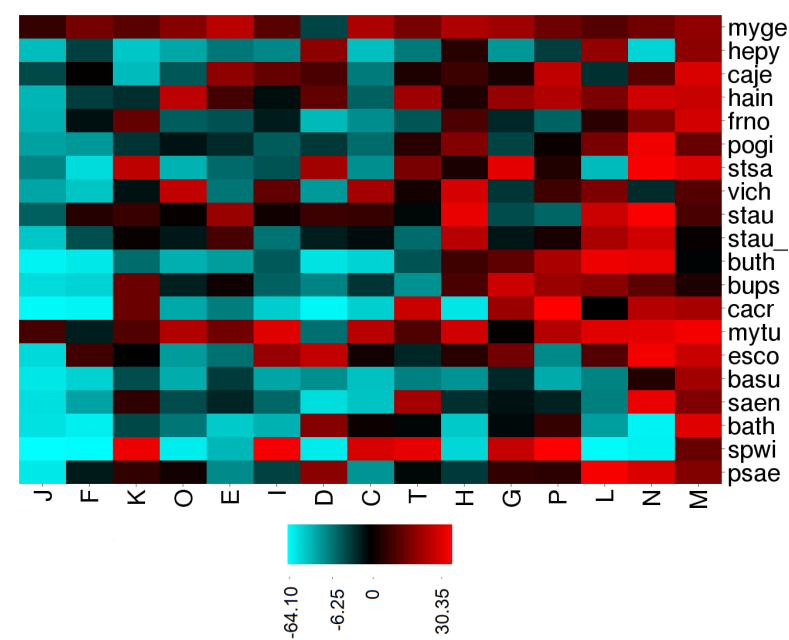

FIG. 6. Z-score of the $K_{a} / K_{s}$ ratios for each COG, with respect to the average value in each bacteria. In the color scale, red means significant positive Z-score (selective pressure), whereas, green indicates significant negative Z-score (purifying pressure). Bacteria are ordered according to their genome size (from top to bottom), while COGs are ordered from left to right according to the ranking of Table IV

\section{Codon bias patterns in bacterial genes}

Previous observations (see Plotkin and Kudla [29] and data therein) point to the fact that each bacterial species has a specific pattern and level of codon bias, which is strongly shared by all its genes; codon bias in specialized categories of genes appears to be just a modulation of the distinctive codon bias of the species. To check this statement, we compute $R S C U$ values of each codon for our set of bacterial genomes, and plot results in Figure 8 - where both codon bias patterns and genomes are clustered according to similarity in the codon usage. The emerging striped structure indicates that these bacteria cluster into at least four groups, characterised by different patterns of codon usage (as measured by $R S C U$ ). Note that this grouping is not driven solely by the GC content of the genomes, as only the first group turns out to be well separated by the others in terms of the group-specific GC content distribution. Overall, this is just a preliminary exploration suggesting that there should be a strong correlation between codon bias patterns of each species and his evolutionary history. Further work is needed, in our opinion, to search for hidden ecological determinants behind this rough classification based on basic codon bias.

Following this line of reasoning, we check whether the

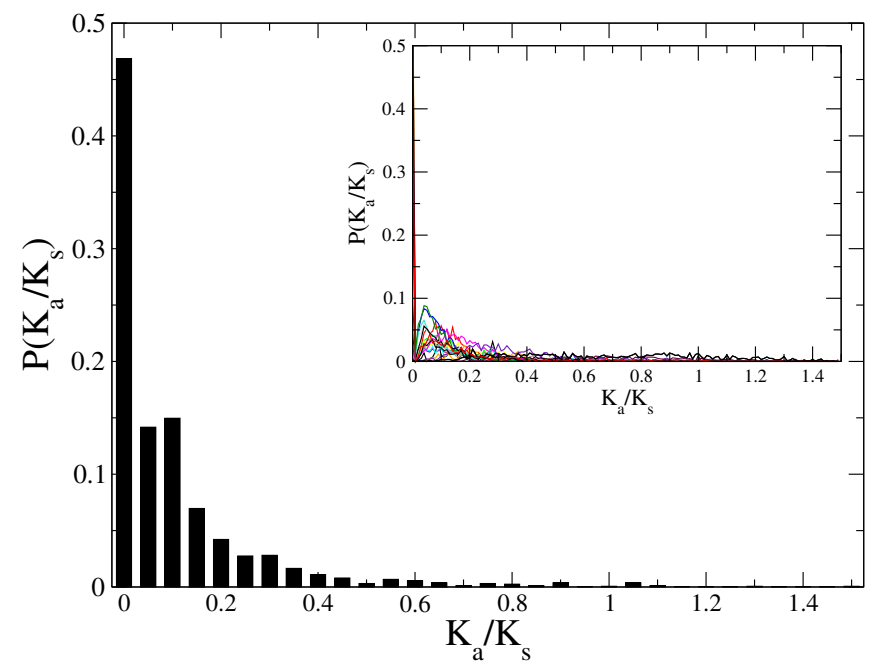

FIG. 7. Frequency distribution of $K_{a} / K_{s}$ for the 83 genes with $\mathrm{ERI}=1$, those having orthologous in all the genomes of the DEG-annotated species of Table I These genes tend to display values of $K_{a} / K_{s}$ concentrated between 0 and 0.2 . Indeed, the average value is 0.085 , the standard deviation is 0.004 and the median is 0.070 , and only in very few cases values bigger than 1 are observed. Inset: same distributions for all genes within individual bacterial genomes

essentiality of a gene has a signature in its codon usage bias. For each of our DEG-annotated genome, we thus compute $R S C U$ values separately for essential and nonessential genes. With the exception of buth and stsa, the two $R S C U$ vectors are very similar for each genome. This indicates that the change in codon bias induced by essentiality, if any, is weak with respect to the prevailing codon bias signature of the species.

\section{Conservation and codon bias of bacterial genes}

In order to investigate whether the codon bias of a bacterial gene is correlated with its degree of conservation, we plot in Figure 9 values of $N_{c}$ (normalised within the species) for genes with given values of ERI. As we did in Dilucca et al. 1] for E. coli, for each bacterial genome it is possible to separate groups of genes with different patterns of codon bias on the basis of their degree of conservation: those with ERI $<0.2$, those with $0.2<\mathrm{ERI}<0.6$ and those with ERI $>0.6$. The evolutionary codon adaptation indeed tends to be higher for genes that are more conserved (genes with ERI $>0.6$ have lower values of $N_{c}$ ). Recall also from Figure 1 that groups of genes with ERI $<0.6$ have a probability of being essential that is less than 0.2 . From these observation, we can conclude that the more a gene is conserved, the more it displays a selected choice of synonymous codons. 

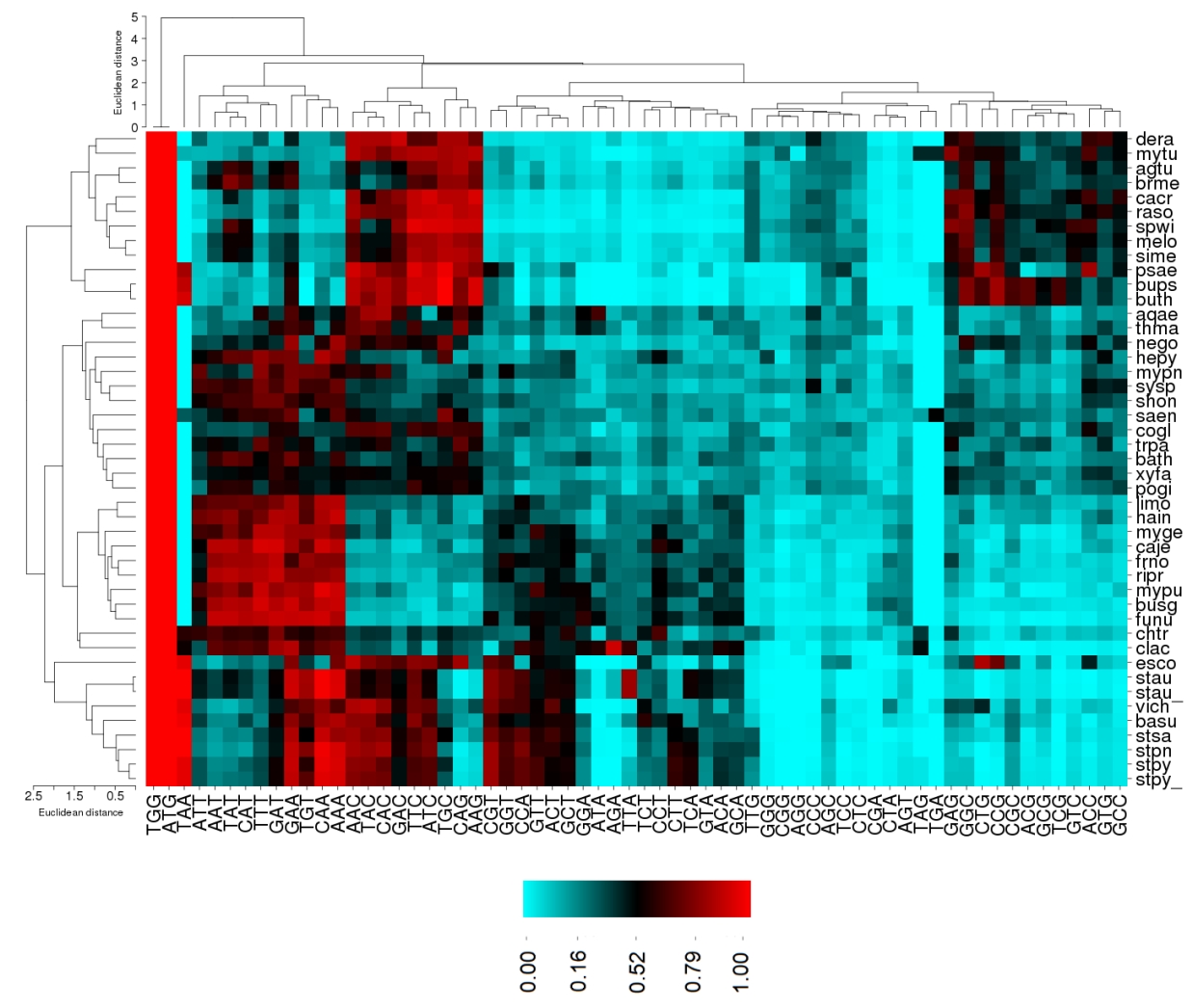
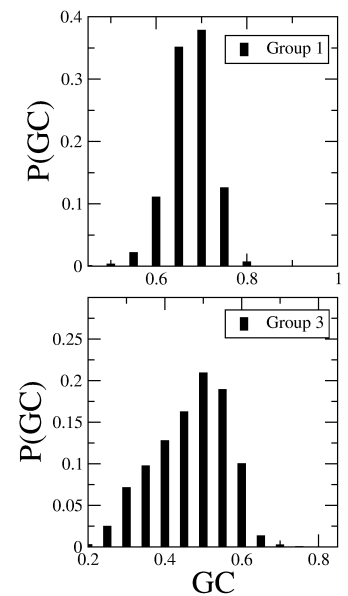
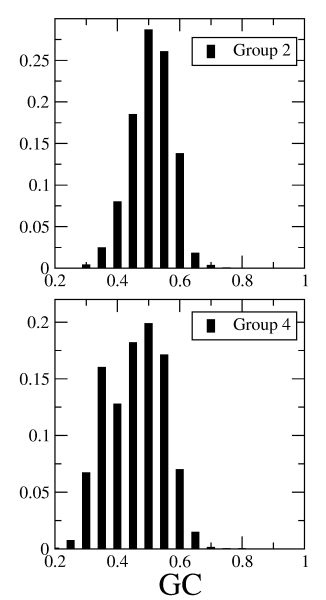

Group 1

Deinococcus radiodurans $\mathrm{R}$ Mycobacterium tuberculosis H37Rv Agrobacterium tumefaciens (fabrum Caulobacter cresentus

Ralstonia solanacearum GMI1000

Sphingomonas wittichii RW1

Mesorhizobium loti MAFF30309

Sinorhizobium meliloti 1021

Pseudomonas aeruginosa UCBPP-PA14 Burkholderia thailandensis E264

\section{Group 3}

Listeria monocytogenes EGD-e

Haemophilus influenzae Rd KW20

Mycoplasma genitalium G37

Campylobacter jejuni

Francisella novicida U112

Rickettsia prowazekii str. Madrid E

Mycoplasma pulmonis UAB CTIP Buchnera aphidicola Sg uid57913
Fusobacterium nucleatum ATCC 25586

FIG. 8. RSCU values of individual codons in different species. Both genomes and groups of codons are clustered by similarity of codon usage. Note that bacterial strains of mypu, shon, stpy and stpy - are missing in the dataset of Luo et al. 8 as well as in the figure. Lower panel: distribution of GC content for genomes in each of the four groups of bacteria listed on the right of the panel. MannWhitney U Tests on the median return 0.05 (group 1 - group 2), 0.003 (group 1 - group 3 ), 0.001 (group 1 - group 4), 0.73 (group 2 - group 3), 0.824 (group 2 - group 4), 0.910 (group 3 - group 4).

\section{Codon bias and evolutionary pressure}

As a conclusive observation, we correlate average $N_{c}$ values with corresponding average value of $K_{a} / K_{s}$ in different bacterial genomes (Figure 10. Bacterial species appear to be separated in at least three clusters, corresponding to different ranges of average values of $N_{c}$, and average values of $K_{a} / K_{s}$ are consistent with the frequency distribution of Figure 7. The few outliers, namely
11 (buth), 13 (cacr) and 19 (spwi), are the species with the highest $K_{a} / K_{s}$ ratios and the lowest $N_{c}$ values: an optimized choice of codons seems to be required to be under a more selective evolutive pressure, remember that lower values of $N_{c}$ indicate more selective choice of synonymous codons. It would be interesting to have data on other bacterial genomes to complete the phase diagram correlating codon bias with evolutionary pressure, of which our Figure 10 is just a preliminary sketch, in or- 


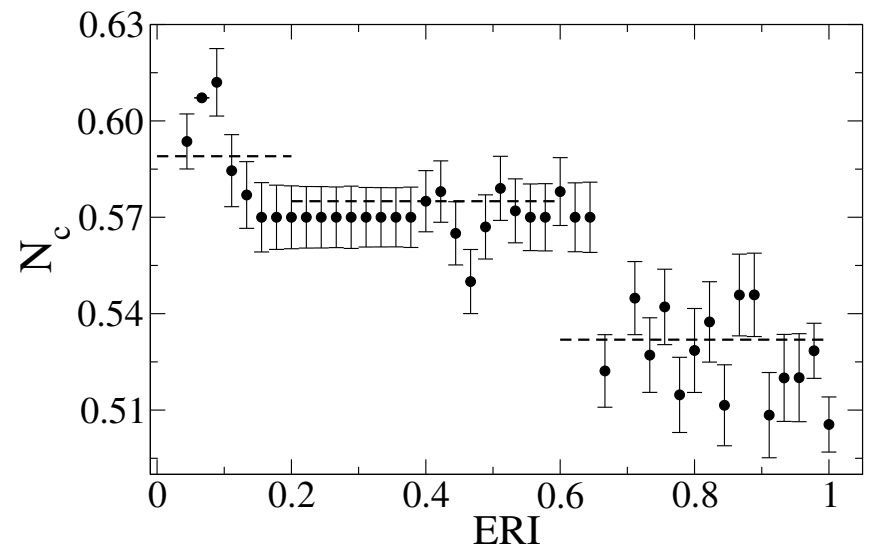

FIG. 9. Codon bias measured by $N_{c}$ for bacterial genes having similar values of ERI. Note that $N_{c}$ values have significantly different averages among bacterial species 30] and thus, for the sake of comparison, they have been normalised within each species between 0 and 1 . The dashed lines represent average codon bias levels of genes in the groups of ERI < 0.2 (specific genes), of $0.2<\mathrm{ERI}<0.6$, and of ERI $>0.6$ (conserved genes).

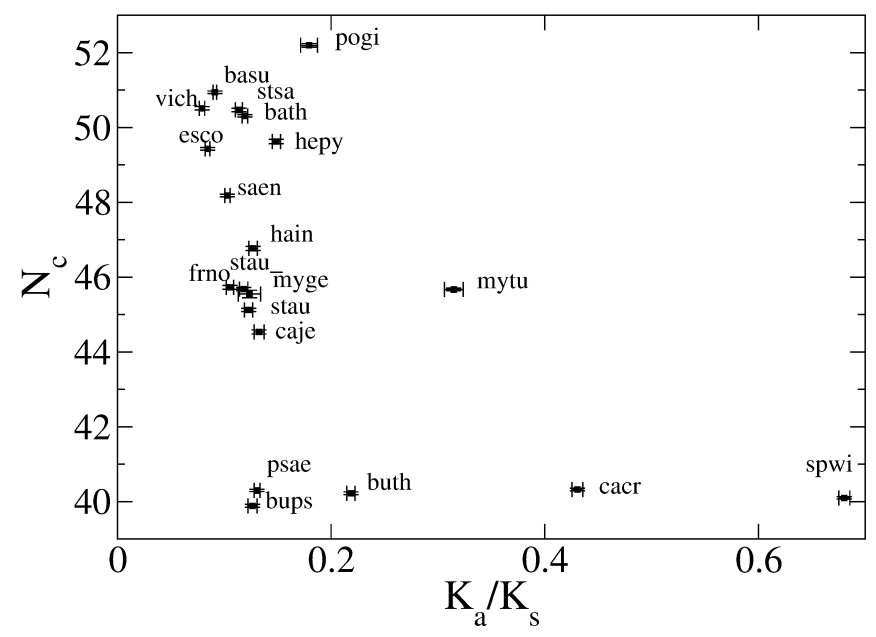

FIG. 10. Average values of (unnormalised) $N_{c}$ and $K_{a} / K_{s}$ for each bacterial species, with error bars denoting root mean square errors.

der to deeply investigate the possibly subtle connection between codon bias at the genetic level with the propensity to mutate at the protein sequence level.

\section{CONCLUSIONS}

Inspired by the results by Luo et al. [8], with this work we further contribute to the elucidation of the intricate connections among gene essentiality, conservation, codon usage bias and evolutionary pressure. In particular, we extended the investigation we performed on E. coli [1] to several bacterial species. That essentiality, conserva- tion, evolutionary pressure and codon bias in bacterial genes, and also in general should be strongly connected is one of those views that are widely shared among life scientist. A view that rests on a broad literature. Our work does not convey radically new messages and the results we have shown largely confirm shared views, but the merit of our observations resides in having shown, carefully, quantitative correlations that have been extended to several genomes. A unified view or theoretical model of the complex interplay among the quantities we have considered here is not yet available. We made a quite unsatisfactory attempt at a ternary representation of codon usage, retention index and selective pressure in the same plot, for essential and non essential genes. The heat maps we get in Figure 11 do not convey any clear emerging pattern and we believe that going beyond binary correlations would require in the future a focused effort by several researchers.

Going back to our findings: as a first result, we have shown that there is a universal exponential correlation between gene essentiality and degree of conservation: genes with high values of the evolutionary retention index (ERI) are more likely to be essential (Figure 1). We have then observed that the number of essential genes is rather conserved among bacterial species. Small bacterial genomes are composed mainly by essential genes but, as the size of the genome increases, the number of nonessential genes increases proportionally (Figure 2). The set of around 500 essential genes in a given bacterial genome is however not composed by genes having orthologs in all the species: essentiality does not imply orthology. This is true also for the core of 83 genes which are strictly retained $(E R I=1)$ in all the species here considered. These genes have a peculiar functional repertoire (mainly COGs $\mathrm{J}$, but also K, L and $\mathrm{O}$, see Table II), and while they are not always essential they have, however, a probability of being essential not less than 0.56 . This set could thus represent an optimal reservoir of potential targets for new antimicrobial components 31.

Regarding functional classification, we have considered how the different clusters of orthologous genes (COGs) accommodate essential and nonessential genes (Figure 3). These two groups turn out to have a complementary spectrum of functions (Table III): essential genes mainly fall into COGs J, M, H, and L, and prevail in functions related to information processing (translation/transcription, replication, recombination and repair), whereas, nonessential genes mainly belong to COGs E, K, G, C and P, with prevalence in metabolic functions (production and transport of energy and basic cellular constituents). Since essentiality implies a certain degree of evolutionary conservation, genes and functions of the first group of COGs should be under a relatively purifying selection, whereas, the second group of functions should be more prone to diversifying selection. Indeed, we have shown in Figures 4 and 5 that more con- 

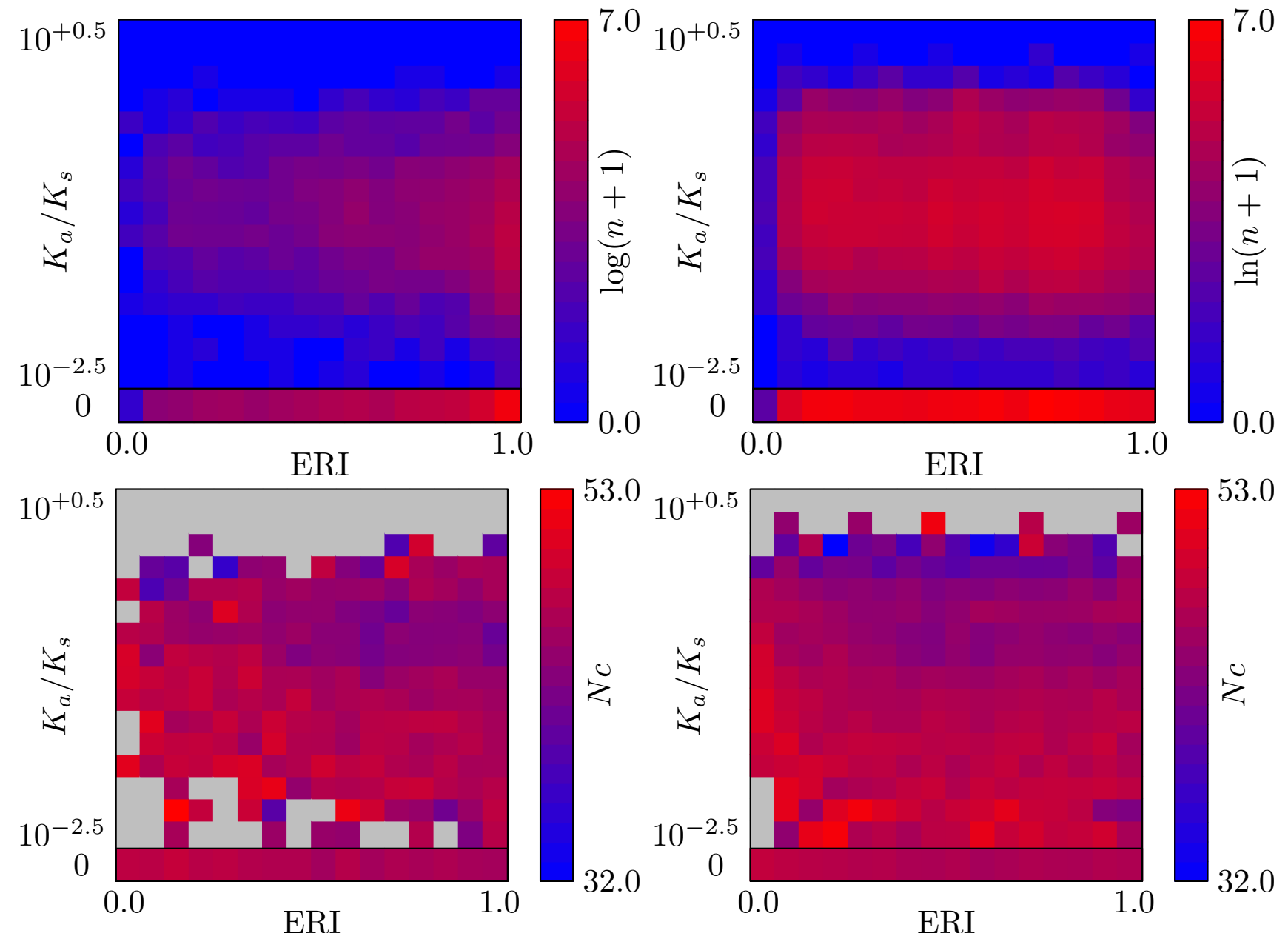

FIG. 11. Heat maps showing the number (upper panels) and average $N_{c}$ value (lower panels) for genes with given values of $E R I$ and $K_{a} / K_{s}$. We consider each genome in our database but split genes into essential and nonessential ones (left and right panels, respectively). Note that the lower (boxed) row of each panel corresponds to $K_{a} / K_{s}=0$, and that in the lower panels empty cells indicate absence of genes. Upper panels do show that the distribution of $K_{a} / K_{s}$ values is highly heterogeneous whatever the ERI values of genes. Lower panels show that $N_{c}$ slightly decreases with increasing ERI, but also seems to decrease for increasing $K_{a} / K_{s}$.

served (shared) genes feature significantly lower values of $K_{a} / K_{s}$ than less conserved genes.

The distribution of $K_{a} / K_{s}$ values of Figure 7 shows that, overall, bacterial genes are under purifying evolutionary pressure, as $K_{a} / K_{s}$ is hardly greater than 1 . Nevertheless, through the relative comparison of the individual Z-scores of $K_{a} / K_{s}$ for genes in different genomes and in different COGs of Figure 6, we could sensibly assess that the different COGs are under different evolutionary pressure and constraints. We have thus proposed a new tentative distinction between a set of relatively more evolutionarily conserved COGs (J, F, K, and O) and a set of more adaptive ones (P, L, N, and M). Such a distinction is clearly at variance with the one coming from the analysis of essential genes we discussed above. Detailing the terms of this contradiction requires further investigation, particularly for understanding the relevance of the coverage of the databases for the consistency between the test based on the COG enrichment in essential genes with that based on the Z-scores of $K_{a} / K_{s}$.

Using $R S C U$ vectors and the effective number of codons $N_{c}$, we have finally shown that it is possible to finely classify bacteria following their codon usage patterns (Figure 8). This classification still requires a consistent interpretation, possibly based on the analysis of ecological relationships among species. We have also shown in Figure 9 that specific and conserved (shared) genes make slightly different use of synonymous codons: more conserved genes have a reduced number of effective codons, a clear indication that conservation of a gene rests on some kind of evolutionary optimization in the use of synonymous codons. Distinguishing essential from nonessential genes does not change the overall classification, indicating that each bacterial species has its own 
strong signature in codon bias. This specificity of the bias suggest where to proceed in the next future, with further investigations on the relevance of codon bias in phylogeny reconstructions and in the prediction of protein-protein interaction networks.

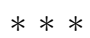

We warmly thank Dr. Hao Luo from the Department of Physics of Tianjin University for sharing with us his evaluations of $K_{a} / K_{s}$ for the genomes in Table I] that are also used by Luo et al. [8].

* maddalena.dilucca@roma1.infn.it

[1] M. Dilucca, G. Cimini, A. Semmoloni, A. Deiana, and A. Giansanti, PLoS ONE 10, e0142127 (2015).

[2] S. Gerdes, M. Scholle, J. Campbell, G. Balazsi, E. Ravasz, M. Daugherty, A. Somera, N. Kyrpides, I. Anderson, M. Gelfand, et al., Journal of Bacteriology 185, $5673(2003)$

[3] G. Fang, E. Rocha, and A. Danchin, Molecular Biology and Evolution 22, 2147 (2005)

[4] C. Peng and F. Gao, Scientific Reports 4, 6001 (2014).

[5] Y. Lin, F. Gao, and C.-T. Zhang, Biochemical and Biophysical Research Communications 396, 472 (2010)

[6] L. D. Hurst and N. G. Smith, Current Biology 9, 747 (1999)

[7] I. K. Jordan, I. B. Rogozin, Y. I. Wolf, and E. V. Koonin, Genome research 12, 962 (2002)

[8] H. Luo, F. Gao, and Y. Lin, Scientific Reports 5, 13210 (2015)

[9] O. Ish-Am, D. M. Kristensen, and E. Ruppin, PLoS ONE 10, e0123785 (2015)

[10] D. Alvarez-Ponce, B. Sabater-Muñoz, C. Toft, M. X. Ruiz-González, and M. A. Fares, Genome Biology and Evolution 8, 2914 (2016)

[11] T. Bergmiller, M. Ackermann, and O. K. Silander, PLoS Genetics 8, e1002803 (2012).

[12] L. D. Hurst, Trends in Genetics 18, 486 (2002).

[13] S. Kanaya, Y. Yamada, M. Kinouchi, Y. Kudo, and T. Ikemura, Journal of Molecular Evolution 53, 290 (2001)
[14] A. Roth, M. Anisimova, and G. M. Cannarozzi, "Measuring codon usage bias," in Codon evolution: mechanisms and models (Oxford University Press Inc. (New York), 2012) pp. 189-217.

[15] A. Dötsch, F. Klawonn, M. Jarek, M. Scharfe, H. Blöcker, and S. Häussler, BMC genomics 11, 234 (2010).

[16] D. A. Benson, M. Cavanaugh, K. Clark, I. KarschMizrachi, D. J. Lipman, J. Ostell, and E. W. Sayers, Nucleic Acids Research 41, D36 (2013)

[17] R. Zhang and Y. Lin, Nucleic Acids Research 37, D455 (2009).

[18] H. Luo, Y. Lin, F. Gao, C.-T. Zhang, and R. Zhang, Nucleic Acids Research 42, D574 (2014)

[19] M. Y. Galperin, K. S. Makarova, Y. I. Wolf, and E. V. Koonin, Nucleic Acids Research 43, D261 (2015)

[20] M. Nei and T. Gojobori, Molecular Biology and Evolution 3, 418 (1986)

[21] F. Wright, Gene 87, 23 (1990).

[22] X. Xia, Molecular Biology and Evolution 30, 1720 (2013)

[23] X. Gong, S. Fan, A. Bilderbeck, M. Li, H. Pang, and S. Tao, Molecular Genetics and Genomics 279, 87 (2008)

[24] R. Gil, F. J. Silva, J. Peretó, and A. Moya, Microbiology and Molecular Biology Reviews 68, 518 (2004)

[25] J. I. Glass, N. Assad-Garcia, N. Alperovich, S. Yooseph, M. R. Lewis, M. Maruf, C. A. Hutchison, H. O. Smith, and J. C. Venter, Proceedings of the National Academy of Sciences 103, 425 (2006).

[26] C. A. Hutchison, R.-Y. Chuang, V. N. Noskov, N. AssadGarcia, T. J. Deerinck, M. H. Ellisman, J. Gill, K. Kannan, B. J. Karas, L. Ma, J. F. Pelletier, Z.-Q. Qi, R. A. Richter, E. A. Strychalski, L. Sun, Y. Suzuki, B. Tsvetanova, K. S. Wise, H. O. Smith, J. I. Glass, C. Merryman, D. G. Gibson, and J. C. Venter, Science 351, aad6253 (2016)

[27] A. Chessher, Protein \& Cell 3, 5 (2012)

[28] B.-M. Koo, G. Kritikos, J. D. Farelli, H. Todor, K. Tong, H. Kimsey, I. Wapinski, M. Galardini, A. Cabal, J. M. Peters, A.-B. Hachmann, D. Z. Rudner, K. N. Allen, A. Typas, and C. A. Gross, Cell Systems 4, 291 (2017)

[29] J. B. Plotkin and G. Kudla, Nature Reviews Genetics 12, $32(2011)$

[30] W. Ran and P. G. Higgs, PLoS ONE 7, e51652 (2012)

[31] F. R. Fields, S. W. Lee, and M. J. McConnell, Biochemical Pharmacology 134, 74 (2017). 